\title{
4
}

\section{Health Care}

\section{General overview}

When it comes to the geopolitics of health, today's developments in strategic technologies present both challenges and opportunities. Improvements and breakthroughs in pharmaceuticals, medical devices, vaccines and patient care have meant improved standards of living and longer life expectancy for much of the world. In certain parts of the world, many infectious diseases have been contained or eliminated, and improvements in sanitation and food safety have reduced the spread of common illnesses, especially in poorer, developing countries.

Nonetheless, the advancing field of health science also leaves much to be desired. Advancements in health-related strategic technologies are improving life in parts of the world, but elsewhere these advances are merely serving to emphasize the ever-growing gap between the world's haves and have-nots. Medical care in the United States and Europe is a marvel of modern technology and innovation, yet much of the world's population still suffers from basic and often treatable diseases. Globally, around one billion people still live in abject poverty with no access to proper health care. What is most frustrating about this situation is how frequently policymakers regard health and security challenges as divergent institutional spheres. ${ }^{1}$ Traditionally, health 'is not immediately perceived as a security issue' but 'recent potential health threats, particularly within the context of food production, travel, and migration, force us to think of health as a paramount security concern'. ${ }^{2}$

Health-related technology depends on the expertise, knowledge and innovation generated by multiple strategic technologies, including biotech, nanotech, and genomics. The types of relevant health technologies available today are seemingly as diverse as the list of existing 
ailments and illnesses. Major health technologies fall into the categories: diagnostic, preventive, therapeutic, auxiliary and rehabilitative. ${ }^{3}$ Some of the most essential health technologies include: diagnostic imaging; medical devices and equipment; blood transfusion; transplantation services; hospital and surgery-related technologies; and laboratory services. ${ }^{4}$ Remarkable achievements in terms of medical devices in the diagnostic domain include imaging technologies (such as Magnetic resonance imagining (MRI) or echography) and electrophysiological methods (such as electrocardiography (ECG) and electromyography (EMG)). ${ }^{5}$ Therapeutic technology examples include lasers, defibrillators, cardiac pacemakers, auditory and visual prostheses and ionizing radiation devices. ${ }^{6}$ Assistive and rehabilitative technological innovations include respirators, braces, hearing aids and eyeglasses. ${ }^{7}$

From a policy perspective, the health care focus needs to be on raising global awareness over transnational health threats, taking preventative measures, and sensitizing people to the increasingly global dimensions of health risks. ${ }^{8}$ A multilateral treaty on disease control requirements and protocols would be a good first step in this direction. ${ }^{9}$

\section{The global health care industry}

In developed countries, costs of health care are rising at a rapid rate. In 2010, total health care expenditure outpaced GDP growth in all OECD countries, reaching an average ratio of 9 per cent of GDP, as opposed to 7.8 per cent in $2000 .{ }^{10}$ Health care spending is expected to increase even more in the foreseeable future.

One of the largest sectors of the industry is the global prescription drug market, which was valued at USD 550 billion in $2006 .{ }^{11}$ Other major sectors include medical equipment and supplies, biotechnology, alternative medicine and health care services. Some of the defining forces of the modern health care industry include increasing the productivity of drugs, hospital equipment and other supplies; the rising costs of health care and the growing number of patients from industrialized countries who are seeking treatment in cheaper, developing countries; a rise in ailments among the elderly, resulting in demand for new types of drugs; and new drug discovery and development especially with regard to genetic sciences. ${ }^{12}$

With global health care costs on a steady upward trend, the overall inclination is for the industry to try to offset its costs, particularly through privatization, higher returns on investment and better management. Governments will maintain their regulatory function in the 
health care industry but, increasingly, they will look to offload the practical provision of health care to more professional organizations. Another industry trend especially important to governments and public health officials is the globalization of disease and addressing the fact that health developments in one corner of the globe can have dramatic impacts on far away populations. ${ }^{13}$ This is true from the perspective of spreading infectious diseases but also in terms of more mundane issues such as the global price of pharmaceutical drugs. ${ }^{14}$ A final industry challenge is the brain drain of medical professionals from developing countries. These talented professionals often leave their home countries in search of better education and opportunities in the industrialized world, thus undermining the quality of medical care at home.

\section{Key technological drivers in health care and medicine}

Genomics, information technology, antimicrobial drugs and nanotechnology are likely to be the key technological drivers in the fields of health care and medicine over the next ten years. Overall trends for the twenty-first century point to a gradual shift to early detection and prevention and to more targeted interventions. ${ }^{15}$ Health science and research will be predetermined by the technical understanding of processes and pathways and the molecular vision of disease. ${ }^{16}$ It will also be more evidence based. ${ }^{17}$ Yet, despite major progress in health science and geopolitics, much work remains to be done. According to the World Health Organization (WHO), in 2030 the key causes of death will be cerebrovascular disease (stroke), ischaemic heart disease, chronic obstructive pulmonary disease and HIV/AIDS. ${ }^{18}$ Major improvements in health technologies will have to be made to face these challenges.

\section{Genomics}

In 2003, scientists finished identifying and sequencing the nearly 25,000 genes in the human genome. ${ }^{19}$ Since then, genomics has rapidly gained momentum and influence in the field of global public health. In many ways, genomics and genetic-related research play a pivotal role in the geopolitics of health care.

For example, in the field of pharmaco-genomics (the process of adjusting therapeutics to the patient's genetic make-up) ${ }^{20}$ and in the related field of pharmaco-proteomics (the use of proteomic technologies in the development of drugs), recent research has focused on tailor-making pharmaceuticals to suit specific genetic profiles. Using knowledge gleaned from genomics, researchers have been able to shape drug design 
around specific disease-causing proteins expressed by genes. ${ }^{21}$ These types of advancement could have positive results for cancer patients, among others. By introducing genes that can stimulate the patient's immune system or make toxins to eliminate tumour cells, genomics will make it possible to fight cancer in a more effective way. ${ }^{22}$

When speaking of genomics, it is important to note that genomicists have sequenced more than just the human genome. In the past decade, researchers have also sequenced the genomes of at least 50 microbial pathogens, and the results of this research may assist in the control of communicable diseases, ${ }^{23}$ helping in the treatment and diagnosis of diseases and potentially limiting the outbreak of new diseases. ${ }^{24}$

Thanks to genomics, many old, expensive health care procedures can now be replaced by cheaper, more accessible ones. Additionally, recombinant vaccines, which bring together biotechnology and genomics, have the potential to be safer and cheaper to produce and store than their traditional counterparts. ${ }^{25}$ Finally, genomics, in combination with information technologies, can make a substantial contribution to health care and medicine. One recent example is the Bio-Linux Computing Platform, a computer program specifically designed to facilitate environmental genomics research. ${ }^{26}$

\section{Information technologies}

Advances in digital and information technologies are playing a key role in spreading scientific research and medical information. Better access to information and improved sharing of medical records and research are some of the most powerful tools for improving global health care. For example, electronic health records make it easier for hospitals to track patients and for non-primary care physicians to access full patient histories. ${ }^{27}$ Static reference information systems like these can be transformed into dynamic decision support systems in hospitals, in private and public sector clinics and at home. ${ }^{28}$ Similarly, the Internet can serve as both an informational resource and a tool to allow doctors to consult with each other in real time, regardless of geographical distances. ${ }^{29}$

Data sharing is an increasingly important component of health care and evidence-based medical research. Current health databases are often small and inadequate for large-scale research, and data is acquired on a project-by project basis. This results in databases that are small, unique, specialized and difficult to navigate and use on a large scale. By combining all this data into streamlined national databases, the health care industry could benefit from rapid learning, a health care technology rooted in IT that would help increase the value of health 
care, monitor health care costs, and track the success of treatment based on the experience of tens of millions of patients being updated in real time. ${ }^{30}$ One of the most successful instances of this is the US National Cancer Institute's cancer Biomedical Informatics Grid (caBIG), an information network that enables members of the cancer community, including researchers, physicians and patients, to share data and knowledge. ${ }^{31}$ The hope is that caBIG will help to accelerate the discovery of new approaches to diagnosing, treating and preventing cancer. ${ }^{32}$ Such technology could easily be applied beyond the realm of cancer therapy, and it has promising potential for improving health care in the twentyfirst century.

Information technology is essential to advancing health care, reducing and containing diseases, and streamlining patient care. There are several existing global communications initiatives designed to manage global health crises such as the outbreak of a new disease. For example, the WHO Global Outbreak Alert and Response Network (GOARN) partnership, created in 2000, uses advanced electronic communication networks and computer applications to 'enhance the network's power in global surveillance and response'. ${ }^{33}$ GOARN, which is maintained by Health Canada, regularly and systematically searches websites, news wires, local online newspapers, public health email services and electronic discussion groups for key words that could signify disease outbreaks. ${ }^{34}$ When a new disease outbreak is identified, GOARN has the resources and contact lists to globally disseminate information to doctors and public health officials.

Overall, the global market for health care information technology is expanding rapidly. It is expected to grow at an annual rate of 13 per cent between 2009 and 2014 and at an annual rate of 16.1 per cent by $2014 .{ }^{35}$ ICT and what has been called 'e-health' have large potential to confront the financial and sustainability challenges the health sector is currently facing. However, many OECD countries still lag behind and government intervention is essential for this potential to be realized. ${ }^{36}$

\section{Antimicrobial drugs}

Antimicrobial drugs are a major technological challenge for global health care. The complexity of pathogens such as HIV and malaria, the slow pace of the development and approval of new antimicrobials and the lack of commercial incentives for new drug development mean that new vaccines and drugs are not keeping pace with fast-evolving, drugresistant pathogens. ${ }^{37}$ 
The central challenge with antimicrobial drugs is their overprescription. Indiscriminate use of antibiotic drugs facilitates microbial resistance to these drugs. As pathogens mutate in response to antibiotics, drugs that were once at the cutting edge of technology quickly become obsolete. For this reason, it can be said that the antibiotic revolution is facing a new range of challenges. ${ }^{38}$ As Professor George Fidas explains:

The first line drug treatment for malaria is no longer effective in over 80 of the 92 countries where the disease is a major health problem. Penicillin has substantially lost its effectiveness against several diseases, such as pneumonia, meningitis and gonorrhea, in many countries. Even vancomycin, the last defense against hospital-acquired infections, is losing its effectiveness. Influenza viruses, in particular, are essentially efficient in their ability to survive and sometimes change into deadly strains. ${ }^{39}$

Ensuring that research and development of new antimicrobial drugs keep up with the rapid evolution of diseases and pathogens will be one of the biggest challenges of global public health in the twenty-first century.

\section{Nanotechnology}

Nanotechnology has the potential to facilitate health care in a number of ways. For example, surfaces enhanced by nanotechnology may eventually be completely bacteria-retardant, thereby facilitating the sterilization of hospitals and other medical facilities. Similarly, nanotech filtration systems could help hospitals improve air quality at a lower cost. ${ }^{40}$ Also, certain medicines could potentially be released into the bloodstream on the nanoscale, thus improving their speed and efficiency. ${ }^{41}$

Public acceptance of the use of nanotechnology, as will be discussed in more details in Chapter 7, is likely to depend on the public's perceptions of its risks and benefits. For this reason, it is important to create a strong legal and ethical framework for the development and application of such innovations. ${ }^{42}$

\section{International regulation}

The WHO is the world's pre-eminent health organization. It is responsible for providing leadership on global health issues, shaping research agendas, setting standards and making policy recommendations, and it sets the tone for global health initiatives ${ }^{43}$ Although most regulatory 
issues like drug approval process or compliance issues are dealt with at the national level, the WHO is the one organization poised to respond to the outbreak of a global epidemic and to comprehensively address transnational health issues such as strengthening the world's health infrastructure and promoting development and health security, especially in the developing world. ${ }^{44}$

To this end, the WHO has established a set of International Health Regulations (IHR), a set of legal principles that are binding on the 194 participating countries around the world. The IHR aims 'to help the international community prevent and respond to acute public health risks that have the potential to cross borders and threaten people worldwide'. ${ }^{45}$ Specifically, the IHR requires countries to inform the WHO about specific disease outbreaks and public health events. This, in turn, sets off a series of preventative measures, such as alert and response. Because of its vast infrastructure, its experience and the diversity of its member countries, the WHO is especially well poised to act in this capacity. ${ }^{46}$

Although the WHO oversees and coordinates global health issues, it has no particular mandate or philosophy that relates specifically to health care technology. ${ }^{47}$ Again, the trend is for these issues to be dealt with at the national or, in some cases, the regional level.

\section{Geopolitical implications of health care technologies}

\section{Infectious diseases}

Naturally occurring diseases have always been a huge threat to humanity, both from a health perspective and an existential one. ${ }^{48}$ In recent years, naturally occurring diseases have been responsible for 25 per cent of deaths worldwide and for 45 per cent in developing countries. ${ }^{49}$ Since the 1970s, 20 maladies have spread or re-emerged in more aggressive forms on the world scene, including highly contagious and deadly diseases such as tuberculosis and malaria. ${ }^{50}$ In addition, at least 30 previously unknown diseases have been discovered (for example Ebola and SARS). The reasons behind the emergence of new diseases and the spread of existing ones are many. For example, the overuse of antibiotics to enhance the growth of chicken and cattle has led to the emergence of more drug resistant microbes. ${ }^{51}$ Additionally, there is increasing human settlement of formerly remote areas such as jungles, and humans have no established immunities to the viruses and bacteria in these areas. Other reasons for the increasing number and growing rates of infectious diseases include the development of megacities in countries with 
poor health infrastructures, an increasingly globalized world with greater opportunities for travel and, consequently, greater numbers of microbial 'hitchhikers' that transfer disease from one region to another, and climate change which is leading to a new distribution and evolution of pathogens. ${ }^{52}$

Obviously, infectious diseases and their spread are a major threat to global public health, but they also influence many other elements of security and geostrategy, including economic strength, national and regional stability and social fragmentation. Sub-Saharan Africa, a region that is unfortunately often cited in this context, has an HIV/ AIDS infection rate of nearly 5 per cent of its population between the ages of 15 and $49,,^{53}$ and the area is reflective of the profound influence that infectious diseases can have on the security and stability of a region. Emerging technologies have the potential to offset some of the gravest challenges posed by these diseases and help to offset their spread. For this reason, emerging strategic health technologies will contribute greatly to global peace and security.

Infectious diseases can take many forms, and whether it is haemorrhagic fevers, AIDS or drug-resistant iatrogenic infections, these are all major health challenges. It is also important to stress that the fight against these infectious diseases must go hand-in-hand with tackling other deadly health problems such as cardiovascular disease, cancers and major chronic, hereditary and non-communicable diseases. ${ }^{54}$

To put infectious diseases into their broader context, it is worth looking at some statistics. Every year, almost two-thirds of child deaths and between a quarter and one-third of all adult deaths worldwide are linked to infectious diseases. ${ }^{55}$ The global incidence of AIDS, tuberculosis and malaria continues to rise, and AIDS and tuberculosis are 'likely to account for the overwhelming majority of deaths from diseases among adults in developing countries by $2020^{\prime} .^{56}$

Strategic technologies are crucial for the purposes of prevention of major infectious diseases. They can help, for example, by preventing HIV transmission due to unsafe blood transfusions. ${ }^{57}$ If the prevention of health care-associated HIV transmission is not improved, unsafe medical injections and blood transfusions could cause up to four million new infections. ${ }^{58}$ Antiretroviral drug technology can change an incurable, fatal disease such as AIDS into a condition that can be managed and dealt with almost like a chronic illness. ${ }^{59}$ This helps infected people to live longer and stronger, meaning less disruption to society. New advances in medicine and medical technology are minimizing the risk of parents spreading HIV to their children, and making these 
technologies cheaper and more widely available should be a top priority for global policymakers.

In the case of an epidemic or pandemic, strategic technologies can play a fundamental role in diagnosing the illness, developing new vaccines, and slowing or stopping the spread of new or evolving viruses. Some of these technologies are medicinal - for example, new vaccines or antiviral medicines - but others merge health technologies with other forms of strategic technology. New technologies played a key role in the detection and fight against the global outbreak of the H1N1 virus in 2009. Airports for instance used infrared heat sensors to monitor the body temperature of all travellers arriving from abroad. Anyone with an abnormal reading had to submit to a medical evaluation to ensure that they were not infected with the potentially deadly virus. ${ }^{60}$

Another example is Veratect, a Seattle-based bio surveillance firm, was able to send early warnings of a potential outbreak to the US authorities ${ }^{61}$ It had identified the potential disease thanks to its information gathering technology tools, which combine artificial intelligence, bio surveillance and the Internet among other things. ${ }^{62}$ Early warning and rapid response systems in general are fundamental elements of preventing global pandemics and ones that are dramatically facilitated by new technologies. Historically, disease surveillance systems have been passive and disease-specific. These numerous individual systems often lack information sharing capabilities and unknown diseases are only detected haphazardly. ${ }^{63}$ Information and communication technologies can help in the surveillance and notification of the spread of infectious diseases.

Genomic technologies can also provide viable future solutions to increasing resistance to viruses. For example, researchers from Geneva and Lausanne, Switzerland, have united their efforts to pioneer 'a technique identifying the gene variants that offer resistance against the [HIV] virus and are passed from generation to generation' ${ }^{64}$ They managed to locate a place where the virus interacted with the immune system of an individual and, as a result, 'to map the susceptibility of one part of the human genome and uncover a family of proteins that had not been previously associated with HIV'. ${ }^{65}$ Technologies like antimicrobial drugs that fight infectious diseases will be invaluable in the fight to improve global public health security.

\section{Global initiatives dealing with public health issues}

Although emerging strategic technologies have great potential to limit the spread and outbreak of disease and illness, getting these technologies 
to the people who need them most is an ongoing global challenge. A number of initiatives exist specifically to match technologies with the neediest populations, and this section describes a few of these in greater depth.

Health care is a strategic challenge that is uniquely suited to global collaboration and public-private cooperation. This fact is reflected in the number of global public health initiatives that encompass representatives from the public and private sectors as well as governmental and non-governmental groups. Some of the most prominent strategic partnerships in the field of global health are the Global Fund to fight AIDS, Tuberculosis and Malaria; the Global Alliance for Vaccines and Immunisation (GAVI) Alliance; the WHO; the Bill \& Melinda Gates Foundation and the World Economic Forum Global Health Initiative.

The Global Fund came into being in January 2002. Its principle mission is to finance national programmes that fight AIDS, tuberculosis and malaria. Funded by over 50 countries from around the world (the largest proportion of the funding comes from the United States), ${ }^{66}$ it has so far committed over USD 18 billion to 136 countries. ${ }^{67}$ The Global Fund is a multi-stakeholder partnership, and although the largest contributors are states, organizations such as the International Olympic Committee and individuals such as the former United Nations Secretary-General, Kofi Annan, also contribute money to the cause. ${ }^{68}$ The Fund's governing bodies include donor and recipient governments, intergovernmental organizations, representatives from non-governmental organizations (NGOs), and the private sector. There are four non-voting members: the government of Switzerland, the Joint United Nations Programme on HIV/AIDS (UNAIDS), the WHO and the World Bank. ${ }^{69}$ The private sector is represented by the Bill \& Melinda Gates Foundation and the McKinsey consultancy. ${ }^{70}$

The diverse nature of the Global Fund allows for unique initiatives that would be unimaginable for a purely governmental group. For example, Bono, the lead singer of the rock band U2, proposed a series of $(\mathrm{RED})^{\mathrm{TM}}$ products designed to engage commercial partners in the fight against disease by getting them to design some of their products with special Global Fund branding. ${ }^{71}$ A portion of the proceeds from these products goes to the fund, thus engaging the public and the private sector in the fight against AIDS, tuberculosis and malaria in a unique (and fashionable) way. ${ }^{72}$

The GAVI Alliance is a public-private partnership, the main objective of which is to improve access to immunization for children in poor countries, thus improving the overall health and social and economic capital 
of some of the world's most impoverished countries. The main members of the alliance are the United Nations Children's Fund (UNICEF), the WHO, the World Bank, the Bill \& Melinda Gates Foundation, developing and donor country governments, research and technical health centres, pharmaceutical and vaccine industry representatives and NGOs. ${ }^{73}$ Interestingly, in contrast to the Global Fund, civil society is significantly outnumbered in the GAVI Alliance by the private and commercial sector. ${ }^{74}$ The GAVI Alliance's 'resources are designed to accelerate the development and introduction of new and underutilized vaccines, enhance injection safety and strengthen routine immunization services as well as the health systems that support immunization and child health care services'. ${ }^{75}$ GAVI works closely with the WHO to assure as much continuity in these vaccines as possible.

In addition to supporting projects like the Global Fund and the GAVI Alliance, the WHO is extremely active in monitoring and developing new health technologies as part of its own organizational mandate. Its Essential Health Technologies division works in the areas of AIDS, malaria and tuberculosis to support the expansion of access to necessary technologies; to provide diagnostic and laboratory support, including prevention and best practices; to promote the strengthening of capacity building to produce positive results at country level, through a dedicated country focus strategy; and to support 'the Millennium Development Goals in reducing child mortality, improving maternal health and combating HIV/AIDS, Malaria and other diseases'. ${ }^{76}$ As the group's website notes, 'access, including in primary health care, to safe and effective health technologies relies on policies for selection and management based on scientific evidence and best practice for organization of their management and use'. ${ }^{77}$

Another major player in the global health scene that is committed to further developing and increasing global access to health technologies is the Bill \& Melinda Gates Foundation. Founded by Microsoft chairman Bill Gates and his wife Melinda, this organization has a wide mandate that covers everything from increasing access to education to finding a cure for AIDS. Overall, the foundation has invested some USD 20.5 million in the study of the genomes and immune systems of the so-called elite controllers, people able to control HIV naturally without drugs. ${ }^{78}$

The World Economic Forum's Global Health Initiative (GHI) was launched in 2001 in Davos, Switzerland: explicitly designed to promote and strengthen private-public partnership in global public health, the initiative supports CEOs from various sectors in their efforts to fight 
HIV/Aids, tuberculosis and Malaria. ${ }^{79}$ According to the World Economic Forum's own figures, GHI and partner initiatives provide access to information, prevention, testing and treatment services for over nine million people. ${ }^{80}$

\section{Health, development and poverty}

Human health is a top security priority and a crucial part of global development and stability. This reality is demonstrated by the fact that the nations that are most vulnerable to illness are often the ones that also suffer from the damaging interaction of poverty and conflict. ${ }^{81}$ Disease reinforces underlying social problems and can sometimes escalate tendencies towards violence. Health problems fuel or foment all sorts of other grave issues, and what is particularly frustrating is that, in most cases, failures in global public health come more from policy shortcomings rather than from a lack of scientific knowledge and resources. The twentieth century was marked by great progress in medical science and human health, but regional inequalities of access to preventive health care and treatments for disease remain and continue to widen. This sad truth is reflected statistically: in developed countries, life expectancy is approximately 80 years or over, whereas in certain sub-Saharan nations it is 40 years or below; and a woman living in a least developed country is 300 times more likely to die in pregnancy or childbirth than a woman from a developed country. ${ }^{82}$

Several initiatives are under way to address this dramatic discrepancy in global health care. In 2000, the then WHO Director-General, Gro Harlem Brundtland, launched the Commission on Macroeconomics and Health. ${ }^{83}$ It was chaired by Jeffrey Sachs and had a mandate to study the links between macroeconomic problems and health issues. ${ }^{84}$ The Commission's aim was to examine the connections between health and poverty and to show that health investment can prompt and accelerate economic growth. ${ }^{85}$ The Commission found that the poor experience greater levels of preventable suffering and death. The lack of clean water, malnutrition and poor sanitation make them more susceptible to disease, and they often lack adequate access to health care. ${ }^{86}$ The epidemics of HIV/AIDS, tuberculosis and malaria greatly affect the socioeconomic structures of most developing countries.

Medical science and knowledge are most often targeted towards the needs of rich nations. ${ }^{87}$ The Global Forum for Health Research estimates that just 10 per cent of global health research resources is spent on the diseases that affect 90 per cent of the world's population: the so-called 
'10/90 gap'. ${ }^{88}$ As the International Task Force on Global Public Goods notes:

Only a small fraction of the worldwide expenditure on health research and development is devoted to the major health problems of the majority of the world's population. Of the 1,233 drugs that reached the global market between 1975 and 1997, only 13 were for tropical infectious diseases that primarily affect the poor. ${ }^{89}$

The private sector of the health care industry is primarily focused on the potential for profit in wealthy countries. ${ }^{90}$ More money is spent per year on finding a cure for baldness, a problem of particular concern to rich, Western men, than on developing a vaccine for malaria. ${ }^{91}$

Health disparities within countries are of just as much concern as inequalities between countries. In the United States, for instance, until recently, the private-public health care system resulted in insufficient access to health care and insurance for large parts of the population. ${ }^{92}$

The health reform legislation adopted by the Obama Administration is expected to lead to significant improvements. On the one hand, the health reform is likely to trigger more regulation and more investment in health technology. On the other hand, it promises to 'make insurance more affordable $[\ldots]$ bring greater accountability to health $[\ldots]$ and end discrimination, ${ }^{, 3}$ thereby covering the basic needs for dignity of many more US citizens. Additionally, it will reduce the cost of health care because more preventive medicine will help impeding diseases.

Over the past decade, the trend in international technology transfers was for 'an increasing role for the private sector and a more static role for the public sector' ${ }^{94}$ Of the USD 73.5 billion spent on health research and development funding in low- and middle-income countries in 1998, only 3 per cent was public sector funding, and a further 8 per cent was private non-profit funding. ${ }^{95}$ By contrast, in the developed world research is mostly financed by the private sector. In 2000 , about 68 per cent of all US research was carried out by the commercial sector. ${ }^{96}$

One challenge in increasing the developing world's access to health care and drugs is concerns over intellectual property rights. Researchers, scientists and private sector firms would be reluctant to invest heavily in new product development if they could not cover the initial investment in their work. As a solution to this problem, the International Task Force on Global Public Goods recommends balancing the developed countries' concerns over intellectual property rights with a multilateral 
agreement on open access to basic science and technology (ABST). ${ }^{97}$ According to the Task Force, the ABST would:

Help resolve the free-rider problems that reduce investments in science and technology relative to a global optimum. Second, it could restrain the tendency of governments to restrict access and to encourage privatization of basic knowledge. This rebalancing of technology development norms in favour of expanding the public domain could help vitalize scientific research in many countries while promoting applied innovation. Third, the agreement could provide an important plank for the construction of modern technological capabilities in poor countries while sustaining access to information for education purposes. ${ }^{98}$

Through an agreement of this type, rich countries would agree to support developing countries in their efforts to generate, assimilate and diffuse knowledge while still profiting from their investments and intellectual capital. ${ }^{99}$

It is important to focus on the health problems affecting the most disadvantaged, poorest people. The list of key challenges includes improving vaccines, creating new vaccines, controlling insect vectors, improving nutrition, limiting drug resistance, curing infection and measuring health status. ${ }^{100}$ Resolving these challenges would require substantial work aimed at understanding which immunological responses ensure protective immunity, creating single-dose vaccines for newborns, creating vaccines that do not need to be refrigerated and can be delivered without needles, controlling insects that are transmitting agents of disease, creating optimal bioavailable nutrients in a single staple plant species and conceiving immunological methods to cure chronic infections. ${ }^{101}$ Visionary scientific research and technological innovation will have to be combined to meet these global challenges.

\section{An overview of key trends and developments in health care technologies}

Across the globe, the challenge of providing quality and affordable health care is a daunting one. Numerous emerging strategic technologies have the potential to help world leaders and communities meet this challenge. Advances in fields such as genomics, ICT, nanotechnology and drug delivery coupled with better medical tools and equipment are gradually making it easier for doctors to detect, treat and prevent many illnesses. In many cases, this is true even for diseases that were 
previously thought to be incurable. Technologies are making it easier to control and stop the spread of infectious diseases, and improved health is closely correlated with stronger development for poorer countries. Nonetheless, while technological progress is accelerating across many facets of the health care industry, problems of inequality are rampant. Developing countries have little or no access to many health care technologies, and often cost is one of the key prohibitive factors. Genetic testing, prescription drugs and antibiotics can be an economic strain even for wealthy countries, and this reality is only magnified for poor countries. Many international and public-private initiatives have been launched to improve the quality of global health care and to correct imbalances in access and research to health care and related technologies. Their investments will hopefully result in further discoveries and developments that will improve global health, an issue that affects literally every person on this planet. 